\title{
A Comparison of Impairment Abstractions by Multiple Users of an Installed Fiber Infrastructure
}

\author{
D. J. Ives ${ }^{(1)}$, S. Yan ${ }^{(2)}$, L. Galdino ${ }^{(3)}$, D. J. Elson ${ }^{(3)}$, F. J. Vaquero-Caballero ${ }^{(1)}$, G. Saavedra( ${ }^{(3)}$, \\ R. Wang ${ }^{(2)}$, D. Lavery ${ }^{(3)}$, R. Nejabati ${ }^{(2)}$, P. Bayvel ${ }^{(3)}$, D. Simeonidou ${ }^{(2)}$ and S. J. Savory ${ }^{(1)}$ \\ (1) Department of Engineering, University of Cambridge, $9 \mathrm{JJ}$ Thomson Avenue, Cambridge, CB3 OFA, UK., \\ (2) High Performance Networks Group, University of Bristol, Tyndall Avenue, Bristol, BS8 1TH, UK. \\ (3) Optical Networks Group, UCL, Torrington Place, London WC1E 7JE, UK. \\ di231@cam.ac.uk
}

\begin{abstract}
We compare three independent impairment abstractions of an installed fibre infrastructure. Abstractions agreed to within 1.3dB despite being obtained from different nodes using different terminal equipment. Validation using a DWDM virtual topology was within 1.4dB. $\odot 2019$ The Author(s)
\end{abstract}

OCIS codes: (060.4510) Optical communications, (060.4256) Networks, network optimization.

\section{Introduction}

Impairment aware abstraction of network elements, applying a performance metric to links, nodes and transceivers, allows the network management system to create virtual networks while respecting the performance constraints. While there have been numerous demonstrations of abstractions for homogeneous scenarios with common terminal equipment [1-4] they do not address issues of interoperability within a heterogeneous network employing alien wavelengths, as may be expected in future optical networks. In the context of abstraction, a key questions is how does the abstracted link performance vary with the location of the observer and with the type of transmission equipment used? This may impact two key use cases of abstraction; a) where the whole network is abstracted from a single central office or b) where the network is abstracted from a limited set of local light paths. In both cases the uncertainty of the abstraction affects the accuracy of performance predictions of future remote light paths.

Here we carrying out the abstraction of an installed fibre network from 3 different access nodes, and using 3 different sets of transmission equipment, employing a generalized noise to signal ratio as the performance metric as this can be simply accumulated along a light path to predict the overall performance [1]. We compare the abstractions and subsequently confirm the effectiveness and robustness against the two use cases by configuring the physical network elements as a virtual unidirectional DWDM three node ring topology. The performance of the transmitted signals was assessed after 1,2 and 3 hops around the ring and includes signals transmitted between different equipment.

\section{Network Test Facility}

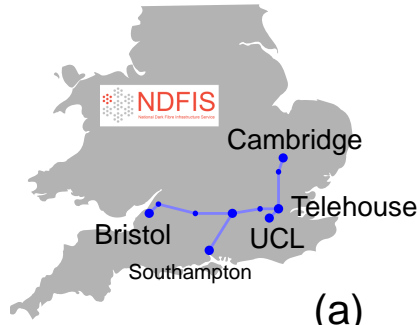

(a)

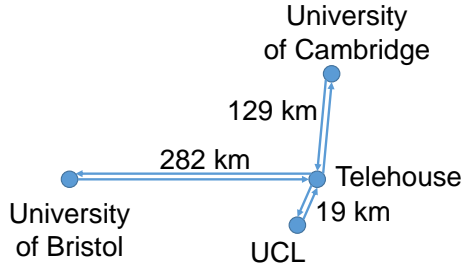

(b)

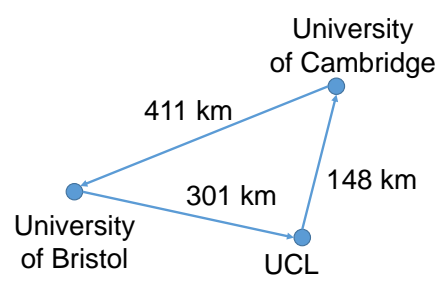

(c)

Fig. 1. The installed NDFIS (a), the configured physical topology (b), and the virtual topology (c).

The UK national dark fiber infrastructure service (NDFIS) is an installed network between the Universities of Cambridge (UoC), Bristol (UoB) and University College London (UCL) as illustrated in Fig. 1a. Each intermediate node location includes EDFA, DCM and a Polatis space switch to allow remote reconfiguration of the fibres and amplifiers. To form a wavelength routed optical network each of the three universities constructed a degree one ROADM to attach to their fiber tails. The ROADM design, transmission and reception capabilities of each partner and the experimental set up are detailed in Fig. 2. The network was physically configured as a star network of three bidirectional links radiating from the space switch at Telehouse as illustrated in Fig. 1b. The launch power for each span was estimated 


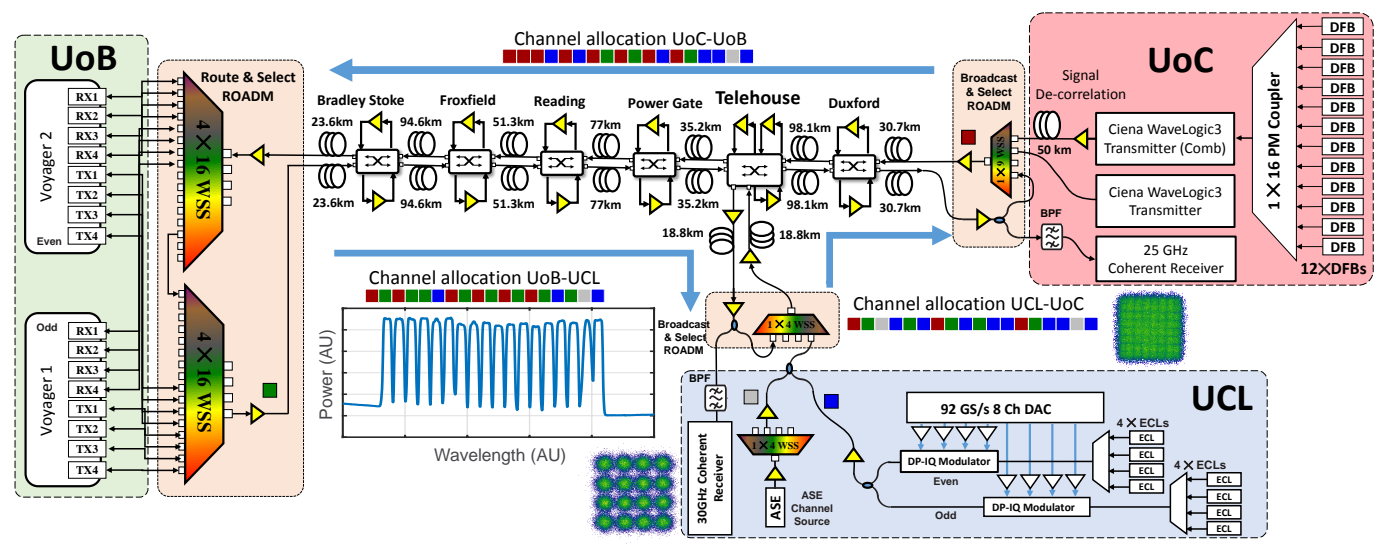

Fig. 2. Experimental configuration, showing NDFIS links, the capabilities of each node, the received spectrum at UCL, 16QAM constellation from UoC to UCL and 64QAM constellation from UCL to UoC. The channel allocation for the 18 channel verification is also displayed where blue, red and green colour represent signals generated at UCL, UoC and UoB, respectively.

from a worst case incoherent GN model but was further constrained by the EFDA capabilities. To maintain wavelength flatness the EDFA must operate at their design gain, as such in some spans the launch power was reduced to avoid saturation of the following amplifier. The launch power into each span was set using the VOA in the Polatis switches.

\section{Network Abstraction}

Each university abstracted the capabilities of the physical links by loading the network with a group of 8 or 9 WDM channels and measuring the performance of the central channel. UoC generated a central $34.5 \mathrm{GBd}$ test channel surrounded by eight $31.5 \mathrm{GBd}$ interference channels, UCL generated a central $32.0 \mathrm{GBd}$ test channels surrounded by 8 interference channels, while UoB transmitted eight $33.1 \mathrm{GBd}$ channels testing all the channels. Each partner measured the performance looped back from their access node to the central node at Telehouse and to the other two partner nodes. UoC and UCL used integrated coherent receivers with real time digital oscilloscopes and off-line DSP to measure the symbol SNR from the received constellation while UoB measured the pre-FEC BER. All measurements were converted to linear noise to signal ratio (NSR) as an abstracted performance metric.

Table 1. Abstracted NSR performance for $1 \mathrm{THz}$ of operational bandwidth, 20 channels. a) of the physical links and $b$ ) of the virtual links.

\begin{tabular}{|l|c|c|c|}
\hline a) & \multicolumn{3}{|c|}{ NSR Abstracted from: } \\
\hline & UoC & UoB & UCL \\
\hline UoC - Thn & 0.0048 & 0.0037 & 0.0045 \\
\hline UoB - Thn & 0.0074 & 0.0082 & 0.0100 \\
\hline UCL - Thn & 0.0029 & 0.0035 & 0.0030 \\
\hline
\end{tabular}

\begin{tabular}{|l|c|c|c|}
\hline b) & \multicolumn{3}{|c|}{ NSR Abstracted from: } \\
\hline & UoC & UoB & UCL \\
\hline Link UoC - UoB & 0.0122 & 0.0120 & 0.0145 \\
\hline Link UoB - UCL & 0.0103 & 0.0118 & 0.0130 \\
\hline Link UCL - UoC & 0.0078 & 0.0072 & 0.0076 \\
\hline
\end{tabular}

The NSR of the loop back from UoC to UoB is, under the assumptions of incoherent Gaussian noise, an accumulation of the NSR of: the back to back (BtB) performance of the transceiver, the ROADM nodes at UoC and UoB and the links. Similarly for the other loop back routes. Given the measured total NSR the NSR of each link, node and transceiver was estimated for the 9 channel transmission. It was assumed that the NSR of each direction of a link were equal. The NSR for each link was increased by $13 \%$ to include the additional NLI for the increased number of channels for the full $1 \mathrm{THz}$ load based on the GN design modelling. Tab. 1a shows the abstracted NSR of the physical links and shows that the different partners estimated the link NSR within 35\% when measured using different transmission signals and from different access nodes.

\section{Virtualised Network Performance}

The physical network was configured as a virtual unidirectional three node ring network between UoC, UoB and UCL as shown in Fig. 1c. From the physical link abstractions the expected NSR for the virtual links was calculated 


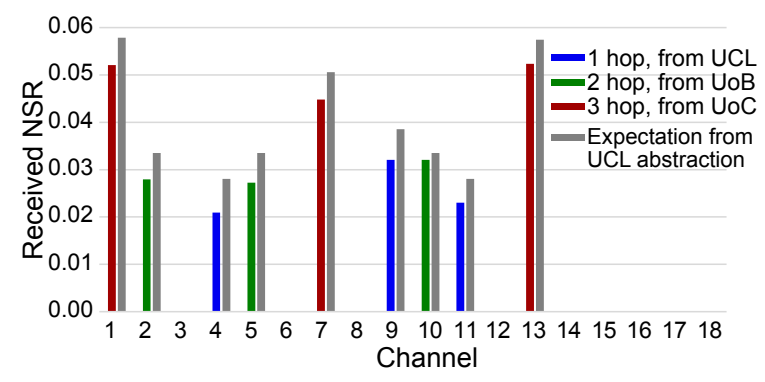

Fig. 3. Received NSR at UoC compared with the expected NSR based on the abstractions from UCL.

as the accumulation of NSR and is shown in Tab. 1b. It can be seen that there is better agreement for the expected NSR between partners along the direct link between them. Where the abstracted link NSR is calculated from indirect measurements the agreement is lower but remains within $26 \%$ or $\approx 1 \mathrm{~dB}$. The channel allocation was designed to give three light paths between each node pair including a full loop of the network from each node and is shown in Fig. 2. 18 channels were fully occupied in each link. Wavelengths were assigned given the experimental restrictions of each partner; 1 three hop route and 1 one hop route were substituted by shaped ASE noise. Reception of signals from other partners was only possible at UoC and UCL using offline receivers.

First, we consider the case where the network has been abstracted from a single location and this is used to predict performance throughout the network. All the light paths were filled with PM-16QAM modulated signals giving $200 \mathrm{~Gb} / \mathrm{s}$ net data rates. Fig. 3 shows the received NSR of the signals at UoC and compares these with the expected performance given the abstraction obtained by UCL. The expected performance is an accumulation of the BtB NSR of the transceivers and the abstracted NSR performance of each link. The measured performance is better than the worst case predicted performance for all light paths. These results suggest that the abstracted NSR can be used to predict light path NSR to within 0.007 or $\approx 1.3 \mathrm{~dB}$. Similarly, the abstraction made from UoC was used to predict the performance of the shortest one hop light path from UCL to UoC. An NSR of 0.0287 was expected allowing a PM-64QAM modulated signal to transmit a net data rate of just above $300 \mathrm{~Gb} / \mathrm{s}$ at $32 \mathrm{GBd}$. The performance of a PM-64QAM modulated signal transmitted from UCL to UoC was measured with an NSR of 0.0267 thus allowing the data rate of $300 \mathrm{~Gb} / \mathrm{s}$. Secondly we used the limited local abstraction from each partner to Telehouse to predict the performance of a three hop light path around the full ring from UCL to UCL. The expected NSR of the links for a full loop was 0.0322 and when combined with the BtB for UCL transceiver gives a predicted light path NSR of 0.0526 while the measured NSR was $38 \%$ better at 0.0380 .

\section{Conclusions}

Three partners have abstracted an installed infrastructure based on a generalized worst case NSR performance metric. A variation of around $1.3 \mathrm{~dB}$ between different transceivers and different access nodes was found for abstractions of the physical links. We have considered the case where a network infrastructure is abstracted from a centralized office and verified this against light paths propagating throughout the network. We also looked at the case where a limited set of light paths are used to abstract local parts of the network and these are combined to create the network wide abstraction. Our experiments suggest that these abstractions are suitable for predicting performance to within $1.4 \mathrm{~dB}$.

Acknowledgements: The authors acknowledge funding support from the UK EPSRC (through the project INSIGHT EP/L026155/2), the UK Royal Academy of Engineering under the Research Fellowships scheme and the EU H2020 project(Metro-Haul,761727), equipment from Ciena and Facebook and use of the UK EPSRC National Dark Fibre Infrastructure Service, NS/A000021/1.

\section{References}

1. D. J. Ives, et al., "Remote Abstraction of an Installed Dark Fiber Network using Noise to Signal Ratio," Proc. OFC, Tu3E.2, (2018).

2. Y. Ou, et al., "Optical Network Virtualisation Using Multitechnology Monitoring and SDN-Enabled Optical Transceiver,' J. Light. Technol., Vol. 36, no. 10, p. 1890, (2018).

3. F. Meng, et al., "Field Trial of Gaussian Process Learning of Function Agnostic Channel Performance Under Uncertainty," Proc. OFC, W4F.5, (2018).

4. S. Oda, et al., "Innovative Optical Networking by Optical Performance Monitoring and Learning Process," Proc. ECOC, Tu3D.3, (2018). 